\title{
Three-Dimensional CST Parameterization Method Applied in Aircraft Aeroelastic Analysis
}

\author{
Hua Su, Chunlin Gong, and Liangxian Gu \\ Shaanxi Aerospace Flight Vehicle Design Key Laboratory, Northwestern Polytechnical University, Xian, Shaanxi 710072, China \\ Correspondence should be addressed to Hua Su; su@nwpu.edu.cn
}

Received 3 March 2017; Accepted 18 June 2017; Published 4 October 2017

Academic Editor: Kenneth M. Sobel

Copyright (C) 2017 Hua Su et al. This is an open access article distributed under the Creative Commons Attribution License, which permits unrestricted use, distribution, and reproduction in any medium, provided the original work is properly cited.

\begin{abstract}
Class/shape transformation (CST) method has advantages of adjustable design variables and powerful parametric geometric shape design ability and has been widely used in aerodynamic design and optimization processes. Three-dimensional CST is an extension for complex aircraft and can generate diverse three-dimensional aircraft and the corresponding mesh automatically and quickly. This paper proposes a parametric structural modeling method based on gridding feature extraction from the aerodynamic mesh generated by the three-dimensional CST method. This novel method can create parametric structural model for fuselage and wing and keep the coordination between the aerodynamic mesh and the structural mesh. Based on the generated aerodynamic model and structural model, an automatic process for aeroelastic modeling and solving is presented with the panel method for aerodynamic solver and NASTRAN for structural solver. A reusable launch vehicle (RLV) is used to illustrate the process for aeroelastic modeling and solving. The result shows that this method can generate aeroelastic model for diverse complex three-dimensional aircraft automatically and reduce the difficulty of aeroelastic analysis dramatically. It provides an effective approach to make use of the aeroelastic analysis at the conceptual design phase for modern aircraft.
\end{abstract}

\section{Introduction}

In the wake of requirements for high lift-drag ratio aerodynamic shape and light-weight structure, the aeroelastic phenomena caused by interaction between fluid and structure have a growing influence on the integrated performance of modern aircraft $[1,2]$. Aeroelastic analysis becomes an important process for modern aircraft design [3,4]. Especially in the conceptual design phase, main performance of an aircraft is determined during this phase and, therefore, how to carry out the aeroelastic analysis quickly and stably to improve the integrated performance and the design rationality of the aircraft scheme will help a lot in the following aircraft design.

Aeroelastic analysis is given attention by lots of researchers [5]. Many tools such as ZAERO [6], ENSAERO [7], and NeoCASS [8] are developed to perform aeroelastic analysis based on the frequency domain analysis method and time domain analysis method and have been widely applied on high aspect ratio wing, unmanned aerial vehicle, and hypersonic aircraft. Although there exist some mature aeroelastic analysis and solving method, the aerodynamic modeling and structural modeling are still a complex and time-consuming process. First, the parametric geometric shape and modeling tools should work together closely and automatically; second, the aerodynamic solver and structural solver should coordinate with each other to ensure that the aeroelastic analysis procedure is executed consistently and accurately. In the conceptual design phase, the design scheme of aircraft usually needs constant modification to improve performance. The size parameters and structural topology of the scheme need to change frequently. It is difficult for the traditional CAD-based geometry modeling method to satisfy the need of rapid geometry iteration and large range modification. Aeroelastic analysis applied in the conceptual design phase faces the following problems:

(1) The aerodynamic model and structural model established in many aeroelastic literatures were complicated; the sizing of the aerodynamic shape and structural layout is difficult and time-consuming, which makes it hard to meet the demand for rapid modification at the conceptual design phase. 
(2) Because of the differences between aerodynamic modeling and structural modeling in mechanism, the aerodynamic mesh and structural mesh are not compatible. The data conversion is used additionally to interchange aerodynamic force and structural deformation between the aerodynamic model and the structural model. Extra computation overhead is needed, and some errors are introduced inevitably.

(3) Quantity and position of the structural inner elements, such as beam, bulkhead, spar, and rib, are hard to be modified using the traditional CAD-based geometry modeling method. The structural layout is vested and cannot obtain the optimal scheme by topology optimization.

These problems hinder the application of aeroelastic analysis in the conceptual design phase. More effective aerodynamic modeling and structural modeling method should be studied to simplify and improve the aeroelastic modeling and analysis process. The CST method is an analytical method developed by Kulfan [9, 10]. It combines a class function representing a specific class of shapes and a shape function defining the deviation from the class function. These provide an efficient shape parameterization ability on complex geometry using fewer design variables. There are some comparisons with other shape parameterization methods $[11,12]$, showing that the CST method has advantages in smoothness, mathematical efficiency, fitting performances, and Intuitiveness. CST has been widely used to parameterize and optimize two-dimensional airfoil $[13,14]$ and simple $3 \mathrm{D}$ aircraft $[15,16]$. Liu $[17,18]$ presents a multiblock CST method to model the hypersonic aircraft with complex shape, which can join adjacent surfaces smoothly and retain the good properties of the CST method. Leal [19] proposes an aerostructural optimization method for determining in a preliminary manner morphing wing configurations that provide benefits during various disparate flight conditions with CST parameterization.

The authors in $[20,21]$ proposed a universal threedimensional CST method for geometry modeling of complex aircraft. It generates complex three-dimensional geometric shape to support various aircraft aerodynamic shape modeling, which gives a simple and effective way to aerodynamic optimization. This novel three-dimensional CST method is extended in aeroelastic analysis of complex aircraft in this article. A novel parametric structural modeling method is proposed based on gridding feature extraction from the aerodynamic mesh generated by the three-dimensional CST method. An automatic and effective way for aeroelastic modeling and analysis is also established. The structure of this article is as follows. First, the basic principle of the three-dimensional CST method is introduced briefly; then the aircraft characteristic components library is established, including fuselage, wing, and empennage; on this basis, the structural modeling method is presented in detail, and the aeroelastic modeling and analysis process is constructed; finally, a static aeroelastic analysis example is used to verify the proposed aeroelastic modeling and analysis process.

\section{Three-Dimensional CST Method}

CST method has an efficient and brief shape description for the two-dimensional airfoils and simple three-dimensional geometries. This section gives an improvement to expand the original two-dimensional CST method to three dimensions. A universal three-dimensional CST method is proposed to provide adjustable parameterization ability to complex threedimensional geometry.

2.1. Basic Cross Section Definition. Most of the aircraft geometry can be described by innumerable cross sections along the axial direction. Combining an analytical function (the class function) with a parametric curve (the shape function), the basic cross section can be defined by the B-spline CST method [22] as follows:

$$
\left.\zeta(\psi)\right|_{\text {upp,low }}=C_{N 2}^{N 1}(\psi) \cdot S(\psi)+\Delta \zeta_{N}(\psi)
$$

where $\psi=z / L_{w} \cdot z$ is the lateral coordinate. $\psi$ is the normalized lateral coordinate. $L_{w}$ is the total length of the basic cross section in the lateral direction. $\zeta(\psi)$ is the normal height in the lateral position. $\Delta \zeta_{N}(\psi)$ is the eccentric distance in the lateral position. $C(\psi)$ and $S(\psi)$ are the class function and the shape function defined in CST method. N1 and $N 2$ are control parameters of the class function. For the symmetrical section, $N 1$ is equal to $N 2$. The class function $C(\psi)$ is defined as follows:

$$
C_{N 2}^{N 1}(\psi)=\psi^{N 1}[1-\psi]^{N 2}
$$

The basic cross section is assembled by the upper $\zeta(\psi)$ and the inverted lower $\zeta(\psi)$. If we set $\Delta \zeta_{N}(\psi)$ to 0 , ignore the shape function, and change the control parameters of the class function simultaneously, different sections can be generated. Various cross section shapes can be generated by changing control parameters of the class function.

2.2. B-Spline Shape Function. The B-spline function can adjust the range of influence by selecting different orders of sub-Bernstein polynomials. It has better local control ability and computation efficiency than Bernstein polynomials. So it has been chosen as the shape function of the CST method in the basic cross section definition. The B-spline function is combined by the massive low-order Bezier curves to approximate the high-order curve. It is defined as follows:

$$
S(\psi)=\sum_{i=0}^{n} b_{i} N_{k}^{i}(\psi),
$$

where $b_{i}, i=0,1, \ldots, n$ is the weight factor. $k+1$ order Bspine basic function is defined as the piecewise polynomials at node vector $T=\left\{t_{0}, t_{1}, \ldots, t_{n+k+1}\right\}, t_{i} \leq t_{i+1} . N_{k}^{i}(\psi)$ is the B-spline basic function defined at $\left\{t_{i}\right\}_{i=0}^{n+k+1}$, which can be 
obtained by recurrence formula De Boor-Cox.

$$
\begin{aligned}
N_{1}^{i}(\psi) & = \begin{cases}1, & t_{i} \leq \psi \leq t_{i+1} \\
0, & \text { otherwise }\end{cases} \\
N_{p}^{i}(\psi) & =\frac{\psi-t_{i}}{t_{i+p+1}-t_{i}} N_{p-1}^{i}(\psi)+\frac{t_{i+p+1}-\psi}{t_{i+p+1}-t_{i+1}} N_{p-1}^{i+1}(\psi) \\
\frac{0}{0} & =0 .
\end{aligned}
$$

The basic cross section defined with B-spline function is as follows:

$$
\left.\zeta(\psi)\right|_{\mathrm{upp}, \mathrm{low}}=C_{N 2}^{N 1}(\psi) \cdot \sum_{i=0}^{n} b_{i} N_{k}^{i}(\psi)+\Delta \zeta_{N}(\psi)
$$

2.3. Three-Dimensional CST Method. The three-dimensional geometry can be considered as a series of original cross sections arranged parallel along the axial direction. By defining proper cross sections using the method mentioned above, we can get an analytical surface to express geometric shape. In order to introduce an analytical axial rule, the coefficient $b$ is replaced by the same B-spline CST definition in formula (5). So the two-dimensional CST function can be expanded to a three-dimensional version, which is shown as follows:

$$
\begin{aligned}
b_{i} & =C_{M 2}^{M 1}(\eta) \cdot S(\eta)+\Delta \zeta_{M}(\eta) \\
S(\eta) & =\sum_{j=0}^{m} b_{j} N_{k}^{j}(\eta),
\end{aligned}
$$

where $\eta=x / L . x$ is the axial coordinate. $\eta$ is the normalized axial coordinate. $L$ is the total length of the generated surface in the axial direction. Bringing formula (6) to formula (5), we have the definition of the three-dimensional analytical surface.

$$
\begin{aligned}
& \left.\zeta(\psi, \eta)\right|_{\text {upp,low }} \\
& =C_{N 2}^{N 1}(\psi) C_{M 2}^{M 1}(\eta) \sum_{i=0}^{n} \sum_{j=0}^{m} b_{i, j} N_{k}^{i}(\psi) N_{k}^{j}(\eta) \\
& \quad+\Delta \zeta_{M, N}(\psi, \eta) .
\end{aligned}
$$

Formula (8) is the analytical expression of the extensional three-dimensional surface, where $\zeta$ is the third-dimensional coordinate value along the $y$ direction based on the twodimensional normalized coordinate $\psi$ and $\eta$. $C(\psi)$ and $C(\eta)$ are the CST's class functions with control parameter $N 1, N 2$, $M 1$ and $M 2 . N(\psi)$ and $N(\eta)$ are the basic functions of the $\mathrm{B}$-spline, which constitute the CST's shape function. $b_{i, j}$ is the discrete control weight factor in the up and low surfaces. Lower case $n$ and $m$ are the orders of B-spline function, which are expressed as the numbers of lateral and axial control points. The total points of the geometric surface are $(n+1) \times$ $(m+1)$. More control points mean more design parameters and better parametric geometric shape design ability. When $n$ $=0$ and $m=0$, the discrete control weight factor $b$ is equal to
1 , the shape function $S(\psi, \eta)$ is also equal to 1 , and then entire geometric surface is controlled by the control factors $N 1, N 2$, $M 1$, and $M 2$ of the class function. $\Delta \zeta_{M, N}(\psi, \eta)$ is the eccentric distance in normal position, and the default value is 0 .

The geometric surface defined in formula (8) can be described as the third-dimensional coordinate value calculated by the two-dimensional mesh points dispersed as the lateral and axial control points. $\psi$ and $\eta$ are the normalized coordinates defined in $[0,1] \times[0,1]$. We also need to define the profile $Z$ in the lateral direction with respect to the axial coordinate $\eta$. The profile $Z$ is expressed the same as the Bspline CST method in formula (5).

$$
Z(\eta)=C_{T 2}^{T 1}(\eta) \sum_{t=0}^{w} b_{t} N_{k}^{t}(\eta)
$$

$b_{t}$ is the discrete control weight factor in the lateral coordinate. From the above definition, we obtain the threedimensional analytical surface, which can be transformed into global Cartesian coordinate as formula (10).

$$
\begin{aligned}
X(\eta)= & \eta \\
Y(\psi, \eta)= & C_{N 2}^{N 1}(\psi) C_{M 2}^{M 1}(\eta) \sum_{i=0}^{n} \sum_{j=0}^{m} b_{i, j} N_{k}^{i}(\psi) N_{k}^{j}(\eta) \\
& +\left.\Delta \zeta_{M, N}(\psi, \eta)\right|_{\text {upp,low }}, \\
Z(\eta)= & C_{T 2}^{T 1}(\eta) \sum_{t=0}^{w} b_{t} N_{k}^{t}(\eta),
\end{aligned}
$$

where $\psi=[0,1], \eta=[0,1]$. The outward boundary box is used to control the size of the geometric surface, and then we get the full design parameters of the entire geometric surface, which includes the following:

$$
\begin{aligned}
& \text { Axial and lateral length: } L, L_{w} \\
& \text { Vertical height: } L_{H 1}, L_{H 2} \\
& \text { Sectional section control factor: N1,N2 } \\
& \text { Vertical section control factor: } M 1, M 2 \\
& \text { Lateral section control factor: T1,T2 } \\
& \text { Surface weight factor: } b_{i, j} \\
& \text { Lateral weight factor: } b_{t}
\end{aligned}
$$

$L, L_{w}, L_{H 1}, L_{H 2}$ are the size parameters of the geometric surface. $N 1, N 2, M 1, M 2, T 1, T 2$ are the control parameters of the class function. $b_{i, j}$ and $b_{t}$ are the discrete control weight factors of the shape function. When $b_{i, j}$ and $b_{t}$ are equal to 1 , the number of total parameters is constant and also minimum. When the $b_{i, j}$ and $b_{t}$ are expressed as matrix, some control points with the same size of the matrix elements are located in the geometric surface to improve the complexity of the geometric shape. So the size of design parameters could be adjusted dynamically. With these weight factors, the three-dimensional CST method has more flexible parametric geometric shape design ability. 


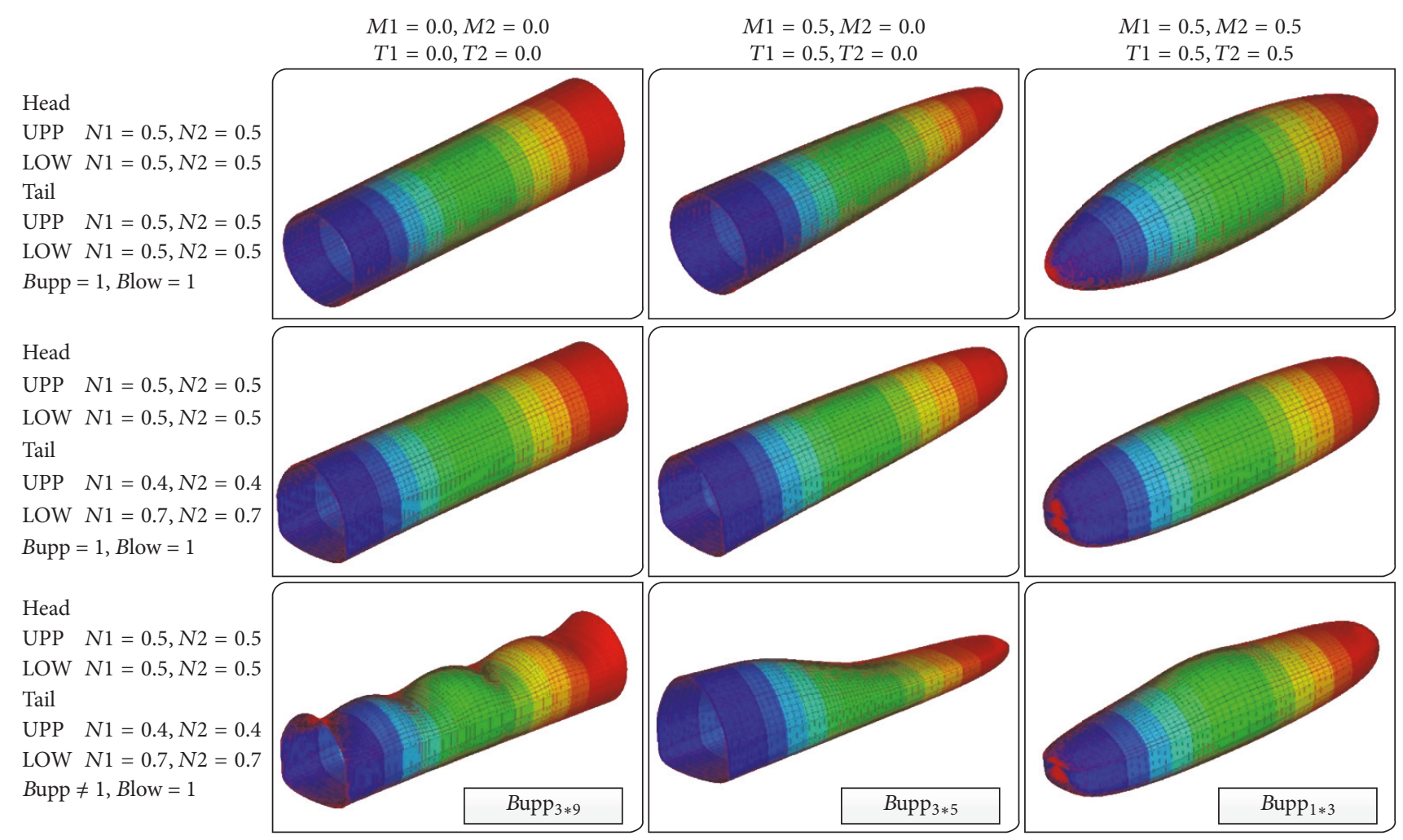

FIGURE 1: Geometric shapes with different control parameters generated by the three-dimensional CST method.

2.4. Basic Geometric Shape. The basic geometric shape is defined by the upper geometric surface and the inverted lower geometric surface. Figure 1 gives some geometric shapes generated by the above three-dimensional CST method with different control parameters. Bupp, Blow, and Bt are the matrix generated by $b_{i, j}$ of the upper surface, $b_{i, j}$ of the lower surface, and $b_{t}$ of the lateral coordinate. The shapes in the same lines are generated with the same value of $N 1$ and $N 2$, but with different $M 1, M 2, T 1$, and $T 2$. The shapes in the same columns are generated with the same values $M 1$, $M 2, T 1$, and T2, but with different N1, N2, and Bupp. The modified shapes in the third line are generated with different sizes of control weight factors of the upper surface compared with the shapes in the second line.

From the various geometric shapes and cross sections shown in Figure 1, the following features can be found for the three-dimensional CST method:

(1) The geometric shape can be expressed as an extruded body made up of a series of basic cross sections with different control factors along the axial direction. The transformation rule of the body is decided by the control factors $N 1_{\text {head }}, N 2_{\text {head }}$ at head and $N 1_{\text {tail }}$, $N 2_{\text {tail }}$ at tail.

(2) The shape profile at the axial and lateral direction is decided by the control factors $M 1, M 2, T 1$, and $T 2$. It allows continuous control of every single basic cross section. When $M$ and $T$ are equal to 0 simultaneously, the corresponding cross section is opened. When $M$ and $T$ are greater than 0 , the corresponding cross section is closed to a point. This feature can be used to generate the closed head and the opening body.

(3) Bupp, Blow, and Bt are matrix with arbitrary size. They produce a global correction or a local adjustment to the geometric shape. The control ability is decided by the size of the matrix.

The shape profile is parameterized by the section control factors, and the geometric shape can be adjusted globally or locally by the weight factor matrix. These features give the three-dimensional CST method a comprehensive parametric geometric shape design ability with adjustable control parameters. Designers could have a more flexible design pattern to parameterize various geometric model.

2.5. Mesh Discretization. A generic mesh discretization method is utilized to generate the corresponding mesh, which will be used as the aerodynamic mesh and structural mesh. The procedure for the three-dimensional CST method is likely to generate structured mesh surface along the $\psi$ and $\eta$ direction. With these characteristics, the surface is dispersed into some control points uniformly. Then a two-dimensional matrix made up of control points is generated, which can be expressed as follows:

$$
\begin{aligned}
& \operatorname{Node}_{\text {upp }}(i, j)=(X(\eta), Y(\psi, \eta), Z(\eta)), \\
& \text { Node }_{\text {low }}(i, j)=(X(\eta), Y(\psi, \eta), Z(\eta)),
\end{aligned}
$$


where $i=1,2, \ldots, M-1, j=1,2, \ldots, N-1 . M$ and $N$ are the numbers of total points in the $\psi$ and $\eta$ direction. The quadrangle is used as the mesh element consisted with these discrete control points

$$
\begin{aligned}
& \text { Element }(t)=[\operatorname{Node}(i, j), \operatorname{Node}(i, j+1), \\
& \text { Node }(i+1, j+1), \operatorname{Node}(i+1, j)] .
\end{aligned}
$$

The $\operatorname{Node}(i, j)$ and Element $(t)$ are used to generate the mesh of the analytical geometric shape. Then we get the corresponding quadrilateral mesh.

\section{Three-Dimensional Parametric Geometry Modeling Method}

With the above three-dimensional CST method, we get the continuous and smooth geometric shape. But single geometric shape cannot provide enough degree of freedom to generate an entire complex aircraft. Usually a complex aircraft can be split into some standard components. So with the component combination method, a universal three-dimensional parametric geometry modeling method is proposed based on the aircraft characteristic components library. The typical aircraft characteristic components include fuselage, wing, and empennage, which are introduced as follows.

\subsection{Aircraft Characteristic Components Library}

3.1.1. Fuselage. The fuselage can be divided into head, headbody, and head-body-tail, three types according to the structural cabins or aerodynamic shape features. By setting the control factors $M 1, M 2, T 1$, and $T 2$ to 1 or 0 , it is easy to generate the semiclosed head and tail, and the opened body. The control factors $N 1$ and N2 must be kept the same value at the joint position between two parts to ensure surface continuation. The detailed control parameters and the three fuselage types are shown in Figure 2(a).

The control factors of the head include

$$
\begin{aligned}
& N_{\text {head }} \\
& =\left[N 1_{\text {head_upp }}, N 2_{\text {head_upp }}, N 1_{\text {head_low }}, N 2_{\text {head_low }}\right] \text {, } \\
& M_{\text {head }} \\
& =\left[M 1_{\text {head_upp }}, M 2_{\text {head_upp }}, M 1_{\text {head_low }}, M 2_{\text {head_low }}\right], \\
& T_{\text {head }}=\left[T 1_{\text {head_upp }}, T 2_{\text {head_upp }}, T 1_{\text {head_low }}, T 2_{\text {head_low }}\right] \text {. }
\end{aligned}
$$

The weight factors of the head include

$$
\begin{aligned}
B & =\left[B_{\text {head_upp }}, B_{\text {head_low }}\right], \\
B_{t} & =\left[B_{t \_ \text {head_upp }}, B_{t \text { _head_low }}\right] .
\end{aligned}
$$

The formulas (13) and (14) are the generic forms of a single part of the component. The others are similar too. By varying these control parameters, various geometric shapes can be generated. The parametric geometric shape design ability is dependent on the size of the matrix $B$ and $B_{t}$. For complex geometric shape, designers can set $B$ and $B_{t}$ to some arbitrary matrix to increase the degree of freedom. But one should note that the number of control parameters also increases as the matrix becomes larger.

3.1.2. Wing. Wing modeling is similar to the fuselage. The control factors $M 1, M 2, T 1$, and $T 2$ are set to 0 to describe the opening tip and root of the wing. The sweepback angle and dihedral angle are also parameterized by mesh offset operation as follows:

$$
\begin{aligned}
& Y_{i, j, \text { correct }}=Y_{i, j}+X_{i, j} * \tan \left(\theta_{y}\right), \\
& Z_{i, j, \text { correct }}=Z_{i, j}+X_{i, j} * \tan \left(\theta_{z}\right) .
\end{aligned}
$$

$\theta_{y}$ is the sweepback angle and $\theta_{z}$ is the dihedral angle. The single wing and double wing are modeled in the components library. The detailed control parameters and the two wing types are shown in Figure 2(b). The tip and root of the wing are open, so the control factors of the cross section only include $N_{\text {in }}$ and $N_{\text {out }}$.

3.1.3. Empennage. Empennage modeling is the same as the wing. The single tail and double tail are modeled in the components library and shown in Figure 2(c).

3.1.4. Others. Some typical components are introduced above, which can be used to create many kinds of common aircraft. There may be other requested components such as engine and nozzle. These can be some simplification of the basic three types components library. For example, the nozzle may be some simplification of the single part fuselage with opening head and tail. For other special components, designers can model it using the three-dimensional CST method and package to the aircraft characteristic components library for reuse.

3.2. Three-Dimensional Geometry Modeling. The aircraft characteristic components library is introduced in Section 3.1. These components are rotated and moved to the proper position to assemble entire complex aircraft. For the discrete control points $P$ of component generated in Section 2.5,

$$
P=\left[\begin{array}{ccc}
x_{1} & y_{1} & z_{1} \\
x_{2} & y_{2} & z_{2} \\
\vdots & \vdots & \vdots \\
x_{n} & y_{n} & z_{n}
\end{array}\right]
$$

The rotation matrix $M_{r}$ and transfer matrix $M_{d}$ are as follows: 


$$
\begin{aligned}
& M_{r} \\
& =\left[\begin{array}{ccc}
\cos \left(\theta_{z}\right) \cos \left(\theta_{y}\right) & \sin \left(\theta_{z}\right) & -\cos \left(\theta_{z}\right) \sin \left(\theta_{y}\right) \\
-\sin \left(\theta_{z}\right) \cos \left(\theta_{y}\right) \cos \left(\theta_{x}\right)+\sin \left(\theta_{y}\right) \sin \left(\theta_{x}\right) & \cos \left(\theta_{z}\right) \cos \left(\theta_{x}\right) & \sin \left(\theta_{z}\right) \sin \left(\theta_{y}\right) \cos \left(\theta_{x}\right)+\cos \left(\theta_{y}\right) \sin \left(\theta_{x}\right) \\
\sin \left(\theta_{z}\right) \cos \left(\theta_{y}\right) \sin \left(\theta_{x}\right)+\sin \left(\theta_{y}\right) \cos \left(\theta_{x}\right) & -\cos \left(\theta_{z}\right) \sin \left(\theta_{x}\right) & -\sin \left(\theta_{z}\right) \sin \left(\theta_{y}\right) \sin \left(\theta_{x}\right)+\cos \left(\theta_{y}\right) \cos \left(\theta_{x}\right)
\end{array}\right], \\
& M_{d}=\left[\begin{array}{lll}
d x & d y & d z
\end{array}\right] .
\end{aligned}
$$

With these matrices, the modified control points $P_{\text {new }}$ are evaluated in formula (18). Then the modified component mesh is generated by these points using the mesh discretization method discussed in Section 2.5.

$$
P_{\text {new }}=P M_{r}+M_{d}
$$

\section{Universal Structural Modeling Method}

Although structural finite element analysis has been widely used in industrial department and research institution, the structure parameterized modeling is still a difficult phase. The most common structural parameterization method is based on parametric CAD model. It is complicated to model complex aircraft and also difficult to support the changing of the structural layout. These limitations cannot satisfy the needs of rapid modification and iterations in the conceptual design phase of the modern aircraft design. This section will provide a detailed illustration of a novel structural modeling method based on the three-dimensional parametric geometry modeling method mentioned above. Structural model is constructed based on the aircraft characteristic components library. Three typical structural models are elucidated below.

4.1. Fuselage. Fuselage is the main body of an aircraft. It is usually designed as a thin-walled structure and constituted by longitudinal stiffeners (like beam and stringer), transverse stiffeners (like bulkhead), and surface skin. A fuselage contains fuel tank, payload, electronic instrument, and other equipment. Also it is as a sole central part connected with wings, empennages, engines, and other components to make up the entire aircraft. The main structural forms of fuselage include girder structure, longeron structure, and monocoque shell structure. Based on the above assumption, all of these structural forms can be simplified as three basic elements: beam/stringer, bulkhead, and skin. The detailed modeling method of these three elements is shown as follows.

4.1.1. Beam/Stringer. Beam and stringer are the longitudinal stiffeners of fuselage structure, used for undertaking axial load coming from fuselage bending, and also used for transferring the load of outer surface skin to bulkhead. Here, the beam and stringer are simplified to one-dimensional beam element. The cross section shape of the beam and the stringer, like I-section, $\mathrm{H}$-section circle-section and so on, can be ignored at the modeling phase and will be considered by the structural solver.
Assuming the surface mesh of fuselage is continuous and compatible among all parts of the component, the discrete nodes of the upper surface or the lower surface can be expressed as

$$
\text { Node }_{\text {Fuselage }}(i, j) \text {, }
$$

where $i=1,2, \ldots, M, j=1,2, \ldots, N . M$ and $N$ are the numbers of control points along the $\psi$ and $\eta$ direction. Vector $B$ is used to locate the position of the beam and the stringer along transverse direction.

$$
B=\left[\begin{array}{llll}
b_{1} & b_{2} & \cdots & b_{n}
\end{array}\right],
$$

where $0 \leq b_{1}<b_{2}<\cdots<b_{n} \leq 1 . B$ is normalized to $[0,1] . b_{k}$ represents the normalized lateral position of the $k$ th beam/stringer. For the discrete nodes, it can be handled as the nearest point $\operatorname{Node}_{\text {Fuselage }}(k, j)$, which has the minimum distance to the $b_{k}$ position in the $j$ th line of the discrete surface consisted of Node Fuselage $(i, j)$. So the node set of the beam/stringer can be defined as

$$
\operatorname{Node}_{B}^{(k)}=\operatorname{Node}(k, j), \quad j=1,2, \ldots, N .
$$

And the element set of the beam/stringer can be defined as

$$
\begin{aligned}
\operatorname{Element}_{B}^{(k)}=[\operatorname{Node}(k, j) & \operatorname{Node}(k, j+1)], \\
& j=1,2, \ldots, N-1 .
\end{aligned}
$$

Then the mesh of beam/stringer can be extracted from the surface mesh of the fuselage. Figure 3 shows the beam/stringer structural layout scheme of a hypersonic aircraft.

4.1.2. Bulkhead. Bulkhead can be divided into three types: normal bulkhead, reinforced bulkhead, and connected bulkhead. It supports the skin of the fuselage to maintain geometric shape and also undertakes some concentrated load. The definition of bulkhead is similar to the beam/stringer. One-dimensional beam element is selected to simplify the bulkhead model. The cross section shape of the beam also is ignored at the modeling phase and will be considered by the structural solver. Vector $F$ is used to locate the position of the bulkhead.

$$
F=\left[\begin{array}{llll}
f_{1} & f_{2} & \cdots & f_{n}
\end{array}\right]
$$



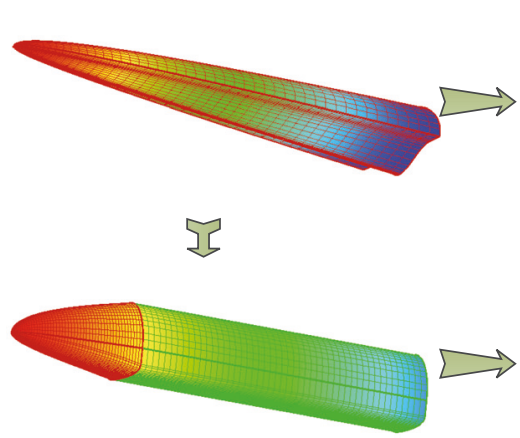

$$
\text { b }
$$

head length, body length, body width, body height

Control factors per component:

$N 1, N 2, M 1, M 2, T 1, T 2$

Weight factors per component: $B i j, B t$

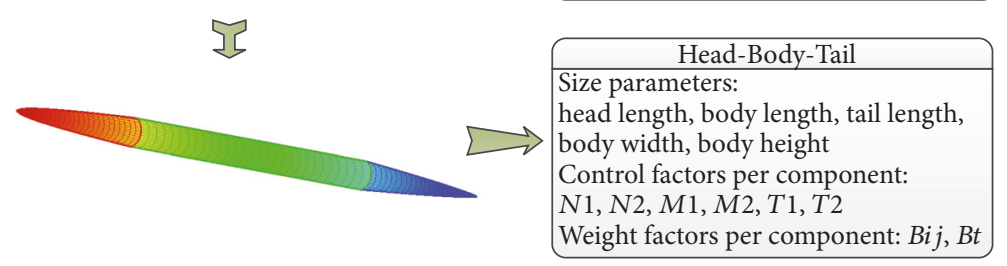

(a)

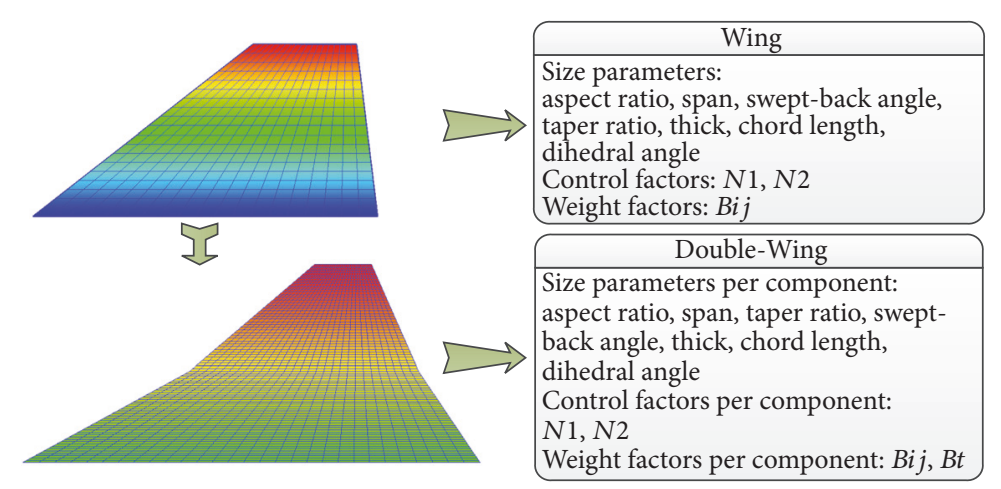

(b)

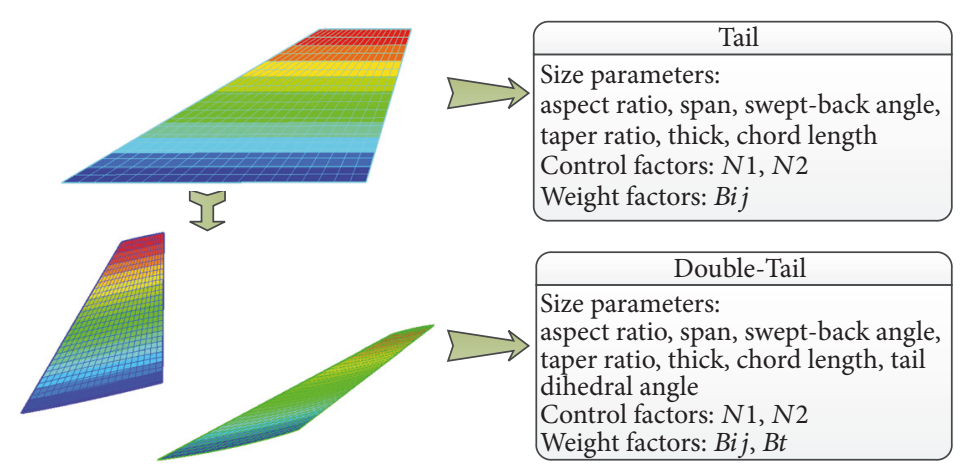

(c)

FIGURE 2: Basic components in the aircraft characteristic components library.

where $0 \leq f_{1}<f_{2}<\cdots<f_{n} \leq 1 . F$ is normalized to $[0,1] . f_{k}$ represents the normalized axial position of the $k$ th bulkhead. For the discrete nodes, it can be handled as the nearest point Node $_{\text {Fuselage }}(i, k)$, which has the minimum distance to the $f_{k}$ position in the $i$ th line of the discrete surface consisting of $\operatorname{Node}_{\text {Fuselage }}(i, j)$. So the node set and the element set of bulkhead can be defined as

$$
\begin{aligned}
& \operatorname{Node}_{F}^{(k)}=\operatorname{Node}(i, k), \quad i=1,2, \ldots, M, \\
& \text { Element }_{F}^{(k)} \\
& =\left[\operatorname{Node}_{\text {Fuselage }}(i, k)\right. \\
& \left.\quad \operatorname{Node}_{\text {Fuselage }}(i+1, k)\right], \\
& i=1,2, \ldots, M-1 .
\end{aligned}
$$




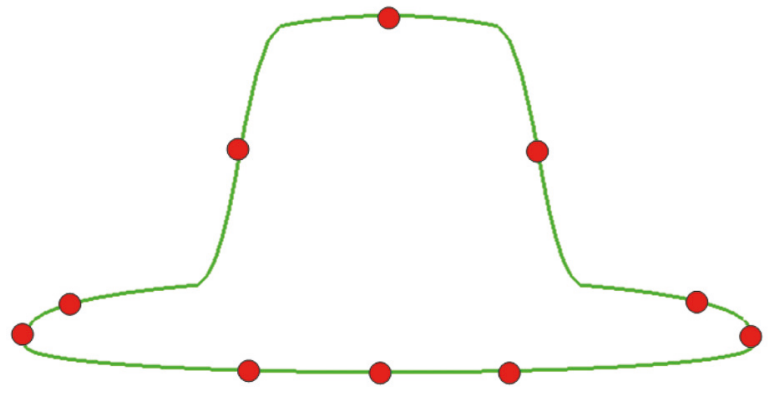

(a) Location points of the beam/stringer

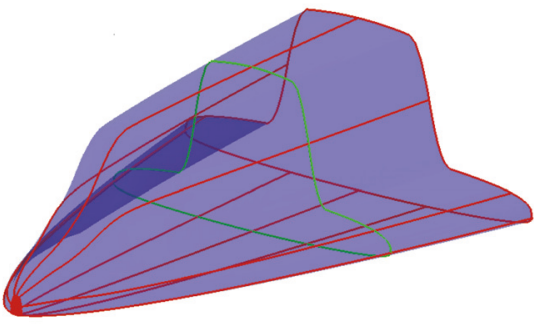

(b) Beam/stringer structural layout scheme

FIgURE 3: The beam/stringer structural layout scheme of a hypersonic aircraft.

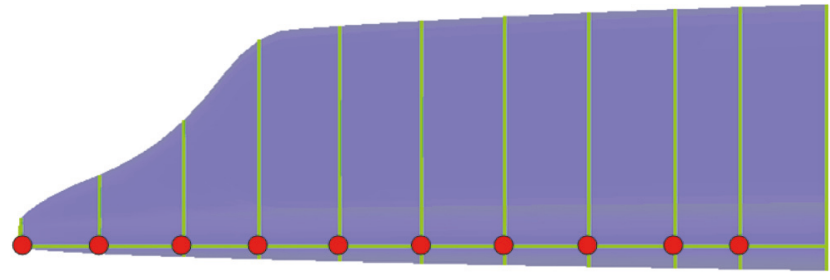

(a) Location points of the bulkhead

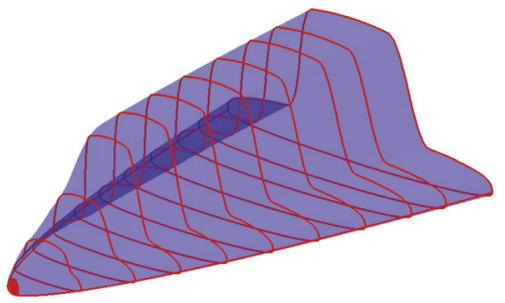

(b) Bulkhead structural layout scheme

FIGURE 4: The bulkhead structural layout scheme of a hypersonic aircraft.

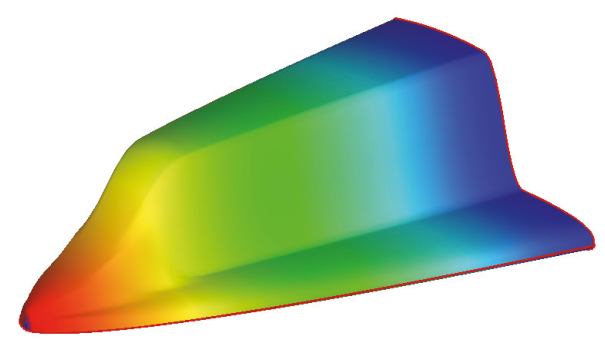

(a) Aerodynamic model of the fuselage

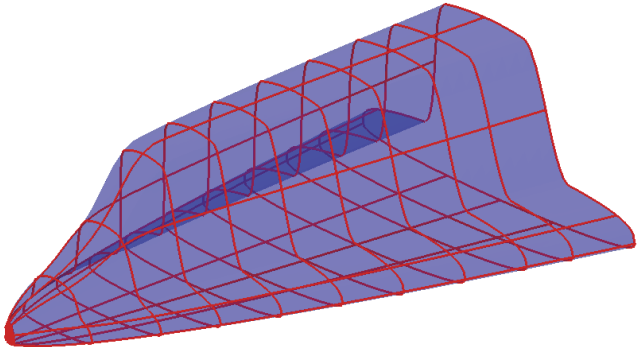

(b) Structural model of the fuselage

FIgURE 5: The aerodynamic model and structural model of a hypersonic aircraft.

Then the mesh of the bulkhead can be extracted from the surface mesh of the fuselage. Figure 4 shows the bulkhead structural layout scheme of a hypersonic aircraft.

4.1.3. Fuselage Skin. Fuselage skin is used to maintain the geometric shape of the fuselage. It should be continuous and smooth to support the aerodynamic solver and structural solver. The discrete surface mesh of fuselage is used for both the structural mesh and the aerodynamic mesh. It has the following advantages: (a) the structural model and the aerodynamic model use the same surface mesh coming from mesh discretization in Section 2.5; the data conversion between these models are no longer needed; (b) the mesh of the beam/stringer and the bulkhead are both extracted from the discrete surface mesh; they are coordinated with surface skin mesh naturally. Based on the above definition, the whole structural model of the fuselage can be generated as Figure 5 .

4.2. Wing. Wing structure consists of skin, spar, stringer, rib, and connector. The following simplifications are used to simplify the wing structural model: (1) the effects of the connectors are ignored; (2) the effects of the flanges of spar, 


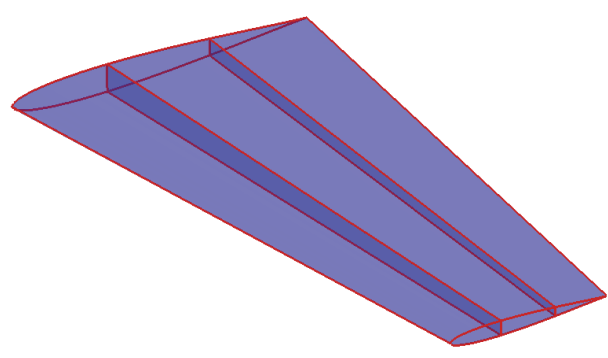

(a) Spanwise structure

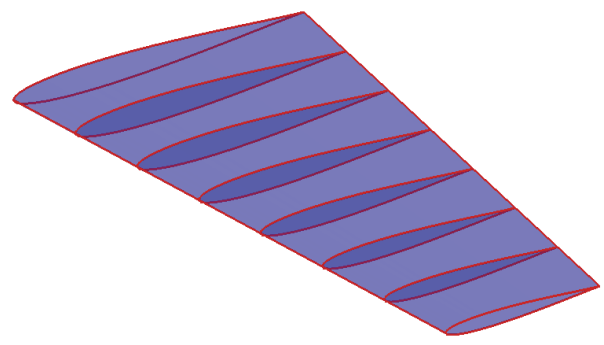

(b) Chordwise structure

FIGURE 6: The spanwise and chordwise structural layout scheme of wing.

stringer, and rib are imputed to the wing skin. Based on these assumptions, the wing structure can be simplified to some two-dimensional shell elements. Three typical basic elements are used to construct the wing structure.

4.2.1. Spanwise Elements. Spanwise elements include spar and stringer. Assuming these elements are arranged along the chordwise direction rigorously. For the discrete nodes $\operatorname{Node}(i, j)$ of the wing, vector $W s$ is used to locate the position of spanwise elements.

$$
W s=\left[\begin{array}{llll}
s_{1} & s_{2} & \cdots & s_{n}
\end{array}\right],
$$

where $0 \leq s_{1}<s_{2}<\cdots<s_{n} \leq 1$. Ws is normalized to $[0,1] . S_{k}$ represents the normalized spanwise position of the $k$ th spanwise elements. For the discrete nodes, it can be handled as the nearest point $\operatorname{Node}_{\text {Wing }}(k, j)$, which has the minimum distance to the $S_{k}$ position in the $j$ th line of the discrete surface consisting of $\operatorname{Node}_{\text {Wing }}(i, j)$. So the node set of spanwise elements can be defined as

$$
\operatorname{Node}_{W s}^{(k)}=\operatorname{Node}_{W i n g}(k, j),
$$

where $j=1,2, \ldots, N . N$ is the number of the spanwise points. $W t$ is used to define the node distribution in the thickness direction.

$$
W t=\left[\begin{array}{llll}
t_{1} & t_{2} & \cdots & t_{s}
\end{array}\right]
$$

where $0 \leq t_{1}<t_{2}<\cdots<t_{s} \leq 1$. Wt is normalized to $[0,1]$ too. The layouts of the upper surface points and the lower surface points are the same. So by arranging some middle points between the upper surface points and the lower surface points by some node distribution, the total nodes of the wing spanwise section can be generated as

$$
\operatorname{Node}_{W s}^{(k)}=\operatorname{linespace}_{W t}^{(k)}\left(\operatorname{Node}_{W \text { supp }}^{(k)}, \operatorname{Node}_{W \text { slow }}^{(k)}\right) .
$$

linespace ${ }_{W t}^{(k)}$ means to arrange points in the regularity of distribution $W t$. The element set can be generated by connecting these nodes. Figure 6(a) shows the spanwise structural layout scheme of wing.
4.2.2. Chordwise Elements. Rib is the chordwise element. Similar to the spanwise elements, vector $W c$ is used to define the position of the rib,

$$
W c=\left[\begin{array}{llll}
c_{1} & c_{2} & \cdots & c_{n}
\end{array}\right]
$$

where $0 \leq c_{1}<c_{2}<\cdots<c_{n} \leq 1$. Wc is normalized to $[0,1]$. The modeling process is the same as the spanwise elements; the node set can be generated as

$$
\operatorname{Node}_{W c}^{(k)}=\operatorname{Node}_{\text {wing }}(i, k),
$$

where $i=1,2, \ldots, M . M$ is the number of the chordwise points. $W t$ is also used to define the node distribution in the thickness direction. So the total nodes of the wing chordwise section can be generated as

$$
\operatorname{Node}_{W c}^{(k)}=\operatorname{linespace}_{W t}^{(k)}\left(\operatorname{Node}_{W c \text { upp }}^{(k)}, \operatorname{Node}_{W c l o w}^{(k)}\right) .
$$

Figure 6(b) shows the chordwise structural layout scheme of wing.

4.2.3. Wing Skin. Similar to the fuselage skin, the discrete mesh of the wing is used for both structural mesh and aerodynamic mesh to maintain consistency. Based on the above definition, the entire structural model of the wing can be generated as Figure 7 .

4.3. Empennage. Structural model of the empennage is the same as the wing. Spanwise elements, chordwise elements, and skin are used to construct the empennage structure.

4.4. Others. Most of the common components have the similar structural layouts to the fuselage and wing. They can be structured by the method mentioned above.

\section{Aeroelastic Modeling and Analysis Process}

The process of aeroelastic modeling and analysis includes three steps: three-dimensional CST modeling, aerodynamic/ structural modeling, and aeroelastic analysis. The process of aeroelastic modeling and analysis is shown in Figure 8, and the detailed illustration is as follows. 


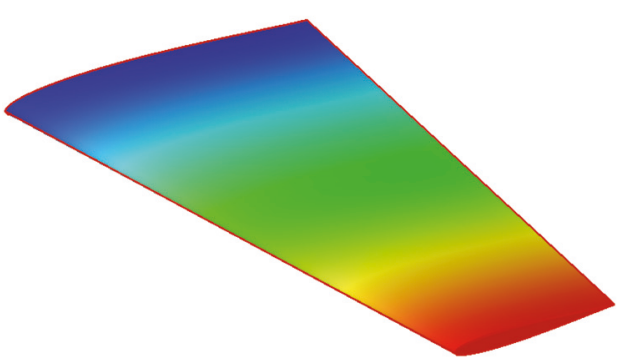

(a) Aerodynamic model

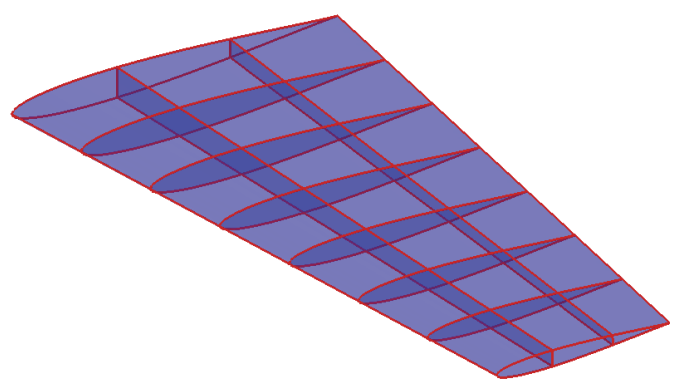

(b) Structural model

FIGURE 7: The aerodynamic model and structural model of wing.

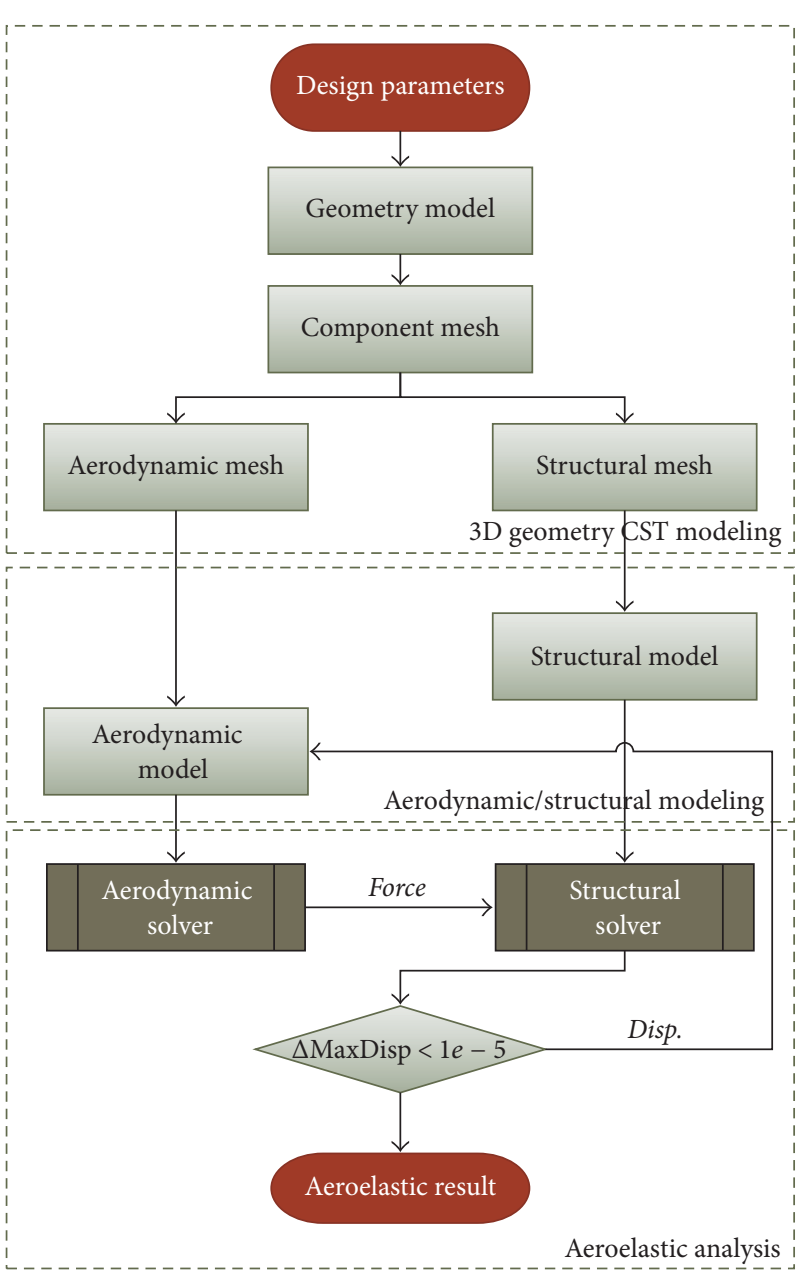

FIGURE 8: The process of aeroelastic modeling and analysis.

5.1. Three-Dimensional CST Modeling. Section 3 lists all design parameters of the three common components. There are four types of design parameters available to use to control the geometric shape: layout parameter, size parameter, shape parameter, and local control parameter. The layout parameter and the size parameter are global design parameters. The former changes the position and the posture of the components, and the latter changes the shape size of the components. They can be used to control the global sizing of aircraft. The shape parameter and local control parameter are local design parameters. The former changes the sketch of the main cross section, and the latter adjusts the details of the component. They can be used to further control the detailed shape of the aircraft. Local control parameter can be any size of matrix. The larger the matrix, the better the parametric geometric shape design ability. According to the requirement of the geometry modeling complexity, the proper design parameters can be selected as the design variables; others may stay constant or vary with respect to the selected parameters. With these design variables, geometry model and corresponding mesh of the component can be generated automatically.

5.2. Aerodynamic/Structural Modeling. A complex aircraft is constituted by several components. Applying the first step, the mesh of these components can be generated, respectively, to make up the entire aircraft. For each of these components, the structural mesh also can be obtained in accordance with the structural modeling method introduced in Section 4. But there are two problems that needed to be solved to generate the integrated aerodynamic/structural model.

The first problem is the redundant mesh caused by the mutual nesting between the connected components. This mesh could influence the precision of aeroelastic analysis. In the worst condition, it may cause some error to the aerodynamic solver and the structural solver. PINPOLYHEDRON, an open-source tool, is used to remove this nested mesh. PINPOLYHEDRON is a $\mathrm{C}++$ code. It provides a novel algorithm to test whether points are inside/outside/on a polyhedron defined by triangular faces and vertices. It can be used for various complicated models such as nonconvex volumes, multimaterial bodies, and so on, and there is no assumption about orientation of the face normal. Above all, the algorithm is very efficient especially for large-scale problems. In this 
research, the fuselage is as the main polyhedron. If a point of other components is detected inside the main fuselage, this point and the relevant mesh are both removed from the component. Looping all points of the component and removing the nested mesh, then the remaining mesh is taken as the valid mesh. This valid mesh is used as the aerodynamic mesh to evaluate the aerodynamic characteristics and is used as the structural mesh to analyze the structural performance.

The second problem is the connection of the relevant components. For aerodynamic analysis, the panel method is used as the aerodynamic solver, so the connection between components is not necessary. For structure analysis, the connection must be modeled to guarantee the force transmission between the connected components, assuming a structural connection exists only between the bulkhead of the fuselage and the beam/spar of other components. The following method is used to generate these structural connections.

(1) For each bulkhead of fuselage, evaluate the midpoint $\mathrm{Pm}_{i}$ by counting the average coordinate of the points set $S m_{i}$ on the bulkhead.

(2) For each beam/spar, find the nearest elements to the fuselage, mark as $E s_{j}$, evaluate the midpoint of $E s_{j}$, and mark as $P s_{j}$.

(3) For each beam/spar, find the nearest bulkhead by $\min _{i}\left|P m_{i}-P s_{j}\right|$, and mark as $P m^{*}$.

(4) Sort the points set $S m^{*}$ on the bulkhead $\mathrm{Pm}^{*}$ by counting the distance from $P s_{j}$, the sorted points set mark as $S m_{r}{ }^{*}$.

(5) The first $N_{r}$ points of the sorted points $S m_{r}{ }^{*}$ are used as the connection points set $S_{1}$. The connection points set of the beam mark $S_{2}$ and $N_{r}$ is the number of the connection points $S_{2}$.

(6) Generate some quadrilaterals by connecting the relevant points between the connection points set $S_{1}$ and connection points set $S_{2}$.

(7) Looping all of the beams of the components, generate nodes set and elements set of the structural connection to obtain the connection mesh.

Figure 9 shows the structural connection between the fuselage and wing generated by the above method. The red line is the bulkheads of the fuselage. The blue line is the beam/stringer of the fuselage. The quadrilaterals with green edges are the structural connection mesh. This method provides a simple way to generate the structural connection mesh between the main fuselage and the connected components.

This structural connection mesh guarantees the grid continuity for force transmission. However, the compelling connection may reduce the quality of these mesh and influence the connection stiffness between the fuselage and the wing. These influences can be corrected by modifying the material attribute or adding a spring element. In this paper, these impacts are ignored temporarily.

5.3. Aeroelastic Analysis. The aerodynamic solver and the structural solver interplay in the aeroelastic analysis process.

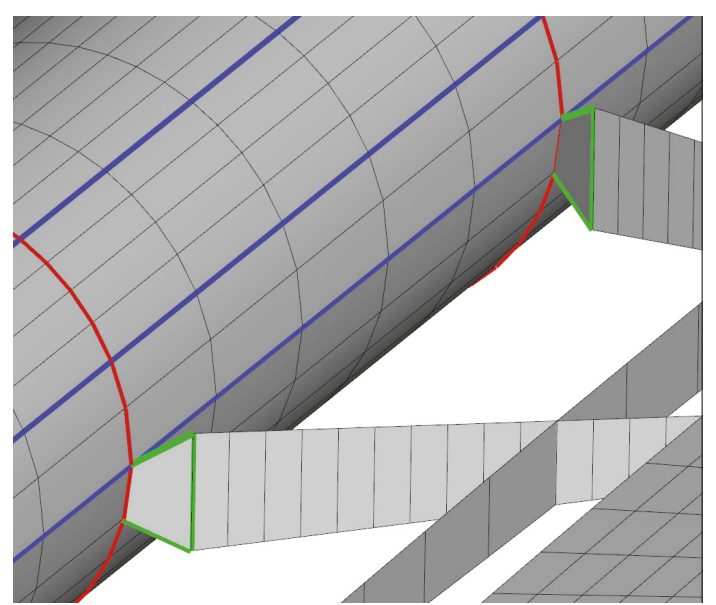

FIGURE 9: The structural connection mesh between the fuselage and the wing.

AeroCalc, a C++ in-house code with the panel method, is used as the aerodynamic solver. The modified Newtonian impact theory is used to evaluate the windward surface, and the Prandtl-Meyer theory is used to evaluate the leeward surface. NASTRAN is used as the structural solver. The structural mesh and control data are written in BDF format as the script file. Every part of the components has its own property section to define thickness, cross section parameters, and material attributes. The outside surface mesh of aerodynamic model and structural model is consistent, so the force obtained from aerodynamic solver can be applied to the structural model and the displacement obtained from structural solver can be applied to the aerodynamic model without any conversion. These simplify the aeroelastic analysis process tremendously.

The three-dimensional CST modeling method is used as a parametric geometry modeling and mesh generation processor to automatically generate aerodynamic and structural model for aeroelastic analysis. The designer can construct an aircraft geometry shape quickly and automatically obtain the corresponding aeroelastic model. An aeroelastic analysis can be carried out quickly and effectively to give a preliminary evaluation of the aeroelastic effect. This could be very helpful for aircraft preliminary design.

The aerodynamic model and structural model use the same surface mesh. The aerodynamic force can be applied to the structural model and the structural displacement can be added to aerodynamic mesh to generate new aerodynamic model directly. This simplification will limit the mesh adaptation for aerodynamic model and structural model, which may increase the computational cost and reduce analysis precision. But it could save the tedious aeroelastic meshing and modeling process, which is time-consuming, especially for complex aircraft. Also these automatic aeroelastic modeling and analysis process can be used for aeroelastic optimization.

\section{Static Aeroelastic Analysis Example}

A reusable launch vehicle (RLV) is used to illustrate the process of aeroelastic analysis. Three basic components, a 


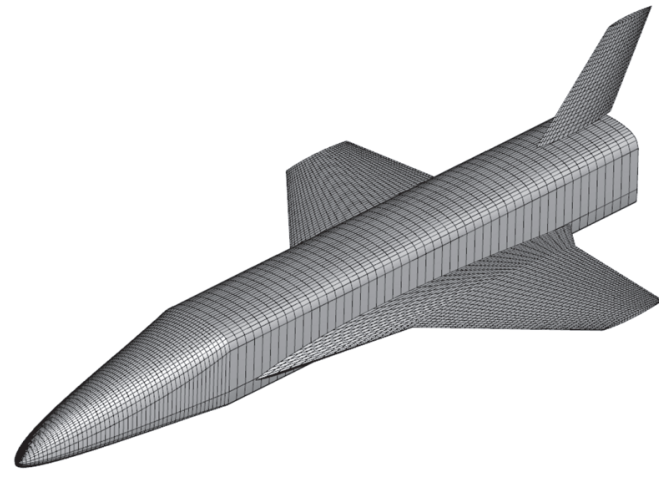

(a) Aerodynamic model

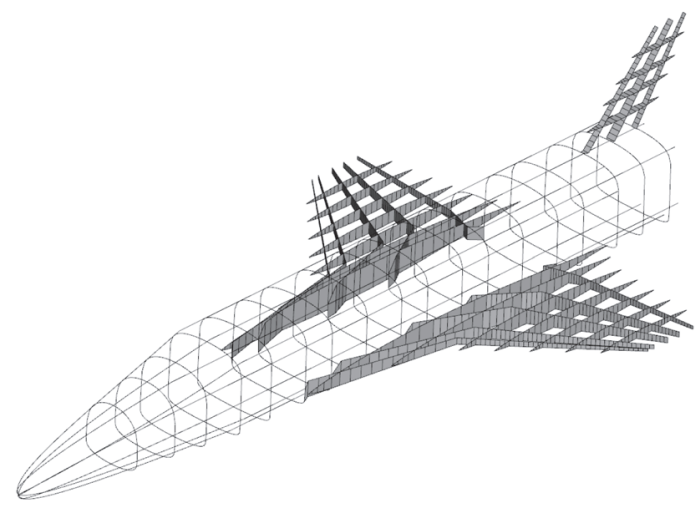

(b) Structural model

FIGURE 10: The aerodynamic model and the structural model of the RLV.

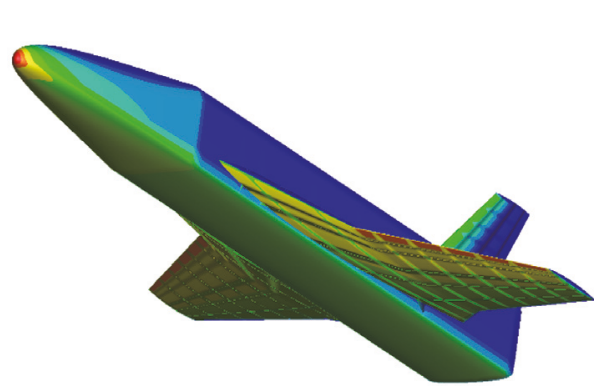

(a) Pressure coefficient distribution

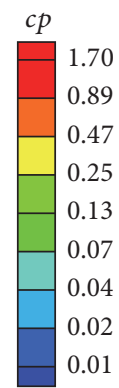

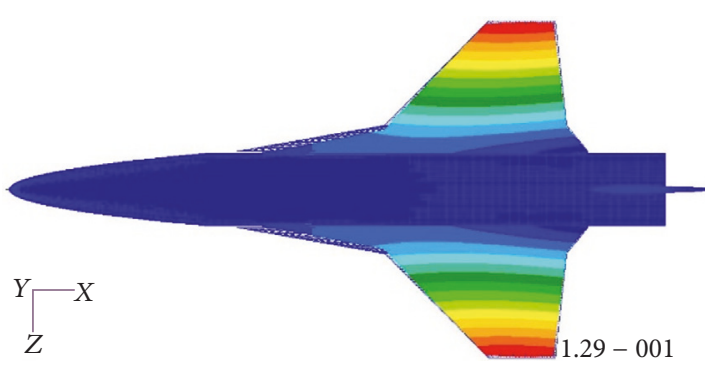

(b) Structural displacement distribution

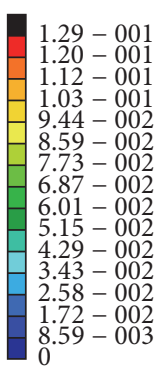

$.59-003$

FIGURE 11: Pressure coefficient distribution and structural displacement distribution at the evaluation points.

head-body fuselage, a double wing, and a tail, are utilized to build up the RLV geometry. The main parameters of the RLV are listed in Table 1, and the trimmed aerodynamic model is shown in Figure 10(a). Eighteen bulkheads and eight beams are arranged in the head-body fuselage. Five spars and seven ribs are arranged in the double wing. Three spars and seven ribs are arranged in the vertical tail. Ten structural connections are used to connect the fuselage and the wing. Three structural connections are used to connect the fuselage and the tail. The integrated structural model is shown in Figure 10(b).

The aluminum alloys are used as structural material for the structural frame and skin, with a density of $2,700 \mathrm{Kg} / \mathrm{m}^{3}$ and an elastic modulus of $72 \mathrm{GPa}$. The total number of the aerodynamic elements is 15506 , which is controlled by the number of points for mesh discretization in the $\psi$ and $\eta$ direction of each surface. The total number of the structural elements is 18691, including the skin mesh, which is the same as the aerodynamic mesh except the nested mesh, the structural component mesh, and the structural connection mesh.

The maximum dynamic pressure trajectory point during reentry is chosen as the aeroelastic evaluation point with $\mathrm{Ma}$ $=3.6$ and $\alpha=6.5$ degree. The pressure coefficient distribution evaluated by the panel method at the maximum dynamic pressure point is shown in Figure 11(a). The corresponding aerodynamic force is applied to the structure surface directly without interpolation. The structural mesh and the displacement distribution are shown in Figure 11(b). The relative error of the maximum deformation in the $Y$ direction is used as the convergence criteria during the aeroelastic iterations and it is set to $1 e-5$.

The aeroelastic analysis of the RLV converges shortly after five iterations. The iteration history of the lift coefficient $\mathrm{Cl}$, the drag coefficient $C d$, and the maximum deformation in the $Y$ direction $d Y$ are shown in Figure 12. In the final converged condition, the $\mathrm{Cl}$ is 1.3 percent lower and the $\mathrm{Cd}$ is 0.37 percent lower, which lead to a 0.94 percent decrease in the lift-to-drag ratio than the initial undeformed condition. The maximum structure deformation in the $Y$ direction occurs at the wingtip position. The maximum deformation at the first structure analysis and the last structure analysis are $0.1287 \mathrm{~m}$ and $0.1271 \mathrm{~m}$. The wingtip deformation is shown in Figure 13. The blue line shows the undeformed wing tip, the orange line shows the deformed wing tip after the first structure analysis, and the red line shows the deformed wing tip at the convergence. The change of the wingtip deformation is 
TABLE 1: Main parameters of the RLV.

\begin{tabular}{lcc}
\hline Components & Design parameters & Value \\
\hline & Head length & $5,000.0 \mathrm{~mm}$ \\
$11,431.0 \mathrm{~mm}$ & $1,821.0 \mathrm{~mm}$ \\
$1,284.0 \mathrm{~mm}$ & Body length & $405.0 \mathrm{~mm}$ \\
\hline Head-body & Body width & $650.0 \mathrm{~mm}$ \\
& Body height upp & $2,600.0 \mathrm{~mm}$ \\
& Body height low & $8,800.0 \mathrm{~mm}$ \\
& Inner wing span length & 1.923 \\
Double wing & Outer wing span length & 2.7 \\
& Inner wing root chord length & $80.0 \mathrm{deg}$ \\
& Inner wing taper ratio & $45.0 \mathrm{deg}$ \\
\hline & Outer wing taper ratio & $1,925.0 \mathrm{~mm}$ \\
& Inner wing sweep back angle & $2,075.0 \mathrm{~mm}$ \\
& Outer wing sweep back angle & 1.724 \\
& Tail span length & $45.0 \mathrm{deg}$ \\
\hline
\end{tabular}

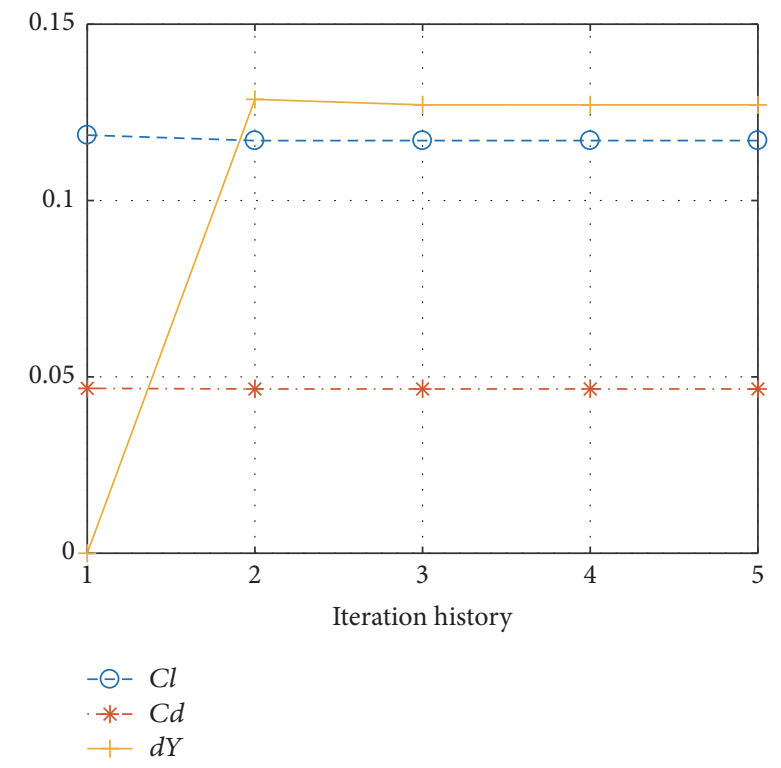

Figure 12: Iteration history of the lift coefficient $\mathrm{Cl}$, the drag coefficient $C d$ and the maximum deformation in the $Y$ direction $d Y$.

not obvious during the aeroelastic iterations, which reaches a stable level after the second step.

The three-dimensional CST parameterization method and the aeroelastic analysis process are written in MATLAB code. The geometry modeling costs less than one second, and the generation of aerodynamic mesh and structural mesh cost $5.5 \mathrm{~s}$. The analysis time of aerodynamic analysis and structure analysis per iteration and the total analysis time are shown in Table 2. The whole aeroelastic modeling and analysis process is very fast and efficient. To generate an entire

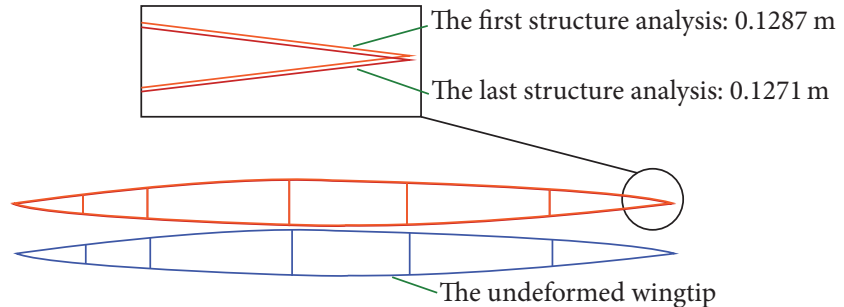

FIgURE 13: The wingtip deformation.

TABLE 2: The cost time of the aeroelastic iterations.

\begin{tabular}{lcc}
\hline & Time per iteration $(\mathrm{s})$ & Total time $(\mathrm{s})$ \\
\hline Aerodynamic analysis & 5.1 & 25.5 \\
Structure analysis & 10.3 & 50.4 \\
Aeroelastic analysis & 16.5 & 82.5 \\
\hline
\end{tabular}

complex RLV and its analysis models only needs several seconds. This will simplify and shorten the aeroelastic analysis and make it easy to use in the aircraft conceptual design phase.

\section{Conclusion and Future Work}

In this paper, a novel aerodynamic and structural modeling method based on the three-dimensional CST is developed to provide a fast and simple way to carry out an entire aeroelastic analysis process. The aerodynamic model is generated by trimming the embedded aircraft characteristic components. The structural model is generated by arranging proper inner parts from the basic mesh topology. The consistency between 
the aerodynamic model and the structural model is satisfied naturally. Finally, an efficient aeroelastic analysis process is created and tested by a RLV. In conclusion,

(1) the three-dimensional parametric geometry modeling method gives a universal way to generate geometry model of common three-dimensional complex aircraft. It inherits the advantages of the original CST method and has fast and stable parametric geometric shape design ability. An aircraft can be modeled with a few control parameters in a few minutes and discretized to surface mesh. More common aircraft characteristic components library will be introduced in future work to give a broader support to various kinds of aircraft;

(2) a universal aeroelastic modeling and analysis process is introduced. By using the three-dimensional parametric geometry modeling method, the aerodynamic model and the structural model can be generated together to keep the mesh consistency. Fluid structure interaction (FSI) can be ignored to simplify the aeroelastic analysis process. This simplification may limit the mesh adaptation for aerodynamic model and structural model, but it gives a fast and simple way to carry out an aeroelastic analysis process for complex aircraft, which is very useful in the conceptual design phase;

(3) the structural modeling method in this article gives an automatic and fast structural layout parameterization and generation approach. Topology of the structure can be parameterized from a wide range stably. This could be used as parameterized structural model for topology optimization;

(4) the generated mesh by the three-dimensional parametric geometry modeling method will be of poor quality at the position where the slope of the surface along axial direction and lateral direction is too large. These are caused by the uniform mesh discretization along the axial direction and lateral direction. These narrow grids may reduce the precision of the aeroelastic analysis. Nonuniform mesh discretization and mesh repairing method will be studied in future work to improve mesh quality.

\section{Conflicts of Interest}

The authors declare that there are no conflicts of interest regarding the publication of this paper.

\section{Acknowledgments}

This research was supported by a fund from the National Natural Science Foundation of China (no. 51505385), the Shanghai Aerospace Science and Technology Innovation Foundation (no. SAST2015010), and the Defense Basic Research Program (no. JCKY2016204B102 and no. JCKY2016208C001). The authors are also thankful to Shaanxi Aerospace Flight Vehicle Design Key Laboratory of NPU.

\section{References}

[1] D. P. Raymer, Aircraft Design: A Conceptual Approach, AIAA Education Series, 4th edition, 2006.

[2] S. A. Brandt, R. J. Stiles, J. Bertin et al., Intorduction to Aeronautics: A Design Perspective, vol. 2nd of AIAA Education Series, 2004.

[3] B. Thuruthimattam, P. Friedmann, K. Powell, and J. McNamara, "Aeroelasticity of a generic hypersonic vehicle," in Proceedings of the 43rd AIAA/ASME/ASCE/AHS/ASC Structures, Structural Dynamics, and Materials Conference, Denver, Colorado, April 2002.

[4] K. K. Gupta, L. S. Voelker, C. Bach, T. Doyle, and E. Hahn, "CFD-based aeroelastic analysis of the X-43 hypersonic flight vehicle," in Proceedings of the 39th Aerospace Sciences Meeting and Exhibit, Reno, Nev, USA, January 2001.

[5] J. Heeg, P. Chwalowski, J. P. Florance, C. D. Wieseman, D. M. Schuster, and B. Perry Jr., "Overview of the aeroelastic prediction workshop," in Proceedings of the 51st AIAA Aerospace Sciences Meeting including the New Horizons Forum and Aerospace Exposition, Grapevine, Tex, USA, January 2013.

[6] D. H. Lee and P. C. Chen, "Nonlinear aeroelastic studies on a folding wing configuration with free-play hinge nonlinearity," in Proceedings of the 47th AIAA/ASME/ASCE/AHS/ASC Structures, Structural Dynamics, and Materials Conference, American Institute of Aeronautics and Astronautics, Newport, Rhode Island, May 2006.

[7] D. Yeh, "Preliminary findings in certification of ENSAERO code for rigid and flexible configuration," in Proceedings of the Fluid Dynamics Conference, American Institute of Aeronautics and Astronautics, Colorado Springs, Colo, USA, June 1994.

[8] C. Luca, R. Sergio, and T. Lorenzo, Neocass: An Integrated Tool for Structural Sizing, Aeroelastic Analysis and MDO at Conceptual Design Level, American Institute of Aeronautics and Astronautics, 2010.

[9] B. M. Kulfan, "Universal parametric geometry representation method," Journal of Aircraft, vol. 45, no. 1, pp. 142-158, 2008.

[10] B. Kulfan, "A universal parametric geometry representation method-'CST'”, in Proceedings of the 45th AIAA Aerospace Sciences Meeting and Exhibit, American Institute of Aeronautics and Astronautics Inc., Reno, Nev, USA, January 2007.

[11] V. Sripawadkul, M. Padulo, and M. Guenov, "A comparison of airfoil shape parameterization techniques for early design optimization," in Proceedings of the 13th AIAA/ISSMO Multidisciplinary Analysis and Optimization Conference (MAO '10), American Institute of Aeronautics and Astronautics, Fort Worth, Tex, USA, September 2010.

[12] S. Nadarajah, P. Castonguay, and A. Mousavi, "Survey of shape parameterization techniques and its effect on threedimensional aerodynamic shape optimization," in Proceedings of the 18th AIAA Computational Fluid Dynamics Conference, American Institute of Aeronautics and Astronautics Inc., Miami, Fla, USA, June 2007.

[13] G. L. Mura and N. Qin, "Local class shape transformation parameterization (L-CST) for airfoils," in Proceedings of the 55th AIAA Aerospace Sciences Meeting, Institute of Aeronautics and Astronautics, Grapevine, Tex, USA, January 2017.

[14] F. Zhu and N. Qin, "Intuitive class/shape function parameterization for airfoils," AIAA Journal, vol. 52, no. 1, pp. 17-25, 2014.

[15] E. D. Olson, “Three-dimensional piecewise-continuous classshape transformation of wings," in Proceedings of the 16th AIAA/ ISSMO Multidisciplinary Analysis and Optimization Conference, 
Institute of Aeronautics and Astronautics, Dallas, Tex, USA, June 2015.

[16] M. H. Straathof and M. J. L. Van Tooren, "Adjoint optimization of a wing using the class-shape-refinement-transformation method," Journal of Aircraft, vol. 49, no. 4, pp. 1091-1100, 2012.

[17] C. Liu, Y. Duan, J. Cai, and J. Wang, "Application of the 3D multi-block CST method to hypersonic aircraft optimization," Aerospace Science and Technology, vol. 50, pp. 295-303, 2016.

[18] C. Liu, Y. Duan, J. Cai, and G. Yang, "Applications of multi-block CST method for quasi-waverider design," in Proceedings of the 52nd Aerospace Sciences Meeting, Institute of Aeronautics and Astronautics, Maryland, Md, USA, January 2014.

[19] P. B. Leal, D. J. Hartl, and C. L. Bertagne, "Aero-structural optimization of shape memory alloy-based wing morphing via a class/shape transformation approach," in Proceedings of the 23nd AIAA/AHS Adaptive Structures Conference, Kissimmee, Fla, USA, January 2015.

[20] S. Hua, G. Liangxian, and G. Chunlin, "The research on geometry modeling method based on three-dimensional CST parameterization technology," in Proceedings of the 16th AIAA/ISSMO Multidisciplinary Analysis and Optimization Conference, American Institute of Aeronautics and Astronautics, Dallas, Tex, USA, June 2015.

[21] H. Su, C.-L. Gong, and L.-X. Gu, "Two-level aerodynamic shape optimization strategy based on three-dimensional CST modeling method," Journal of Solid Rocket Technology, vol. 37, no. 1, pp. 1-6, 2014.

[22] M. H. Straathof and M. J. L. van Tooren, "Extension to the class-shape-transformation method based on B-splines," AIAA Journal, vol. 49, no. 4, pp. 780-790, 2011. 


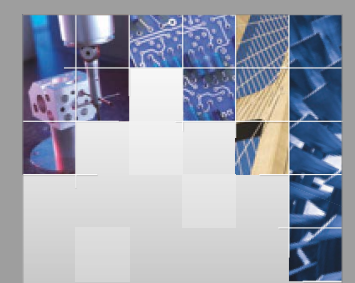

\section{Enfincering}
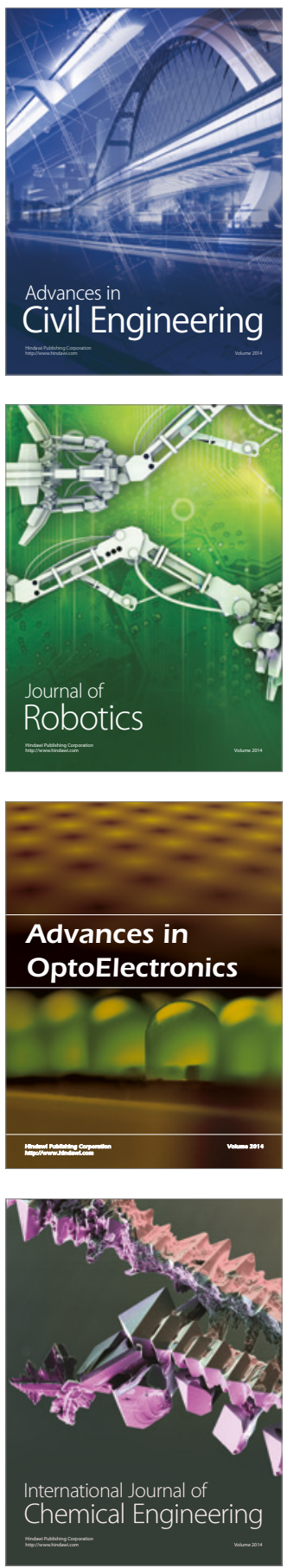

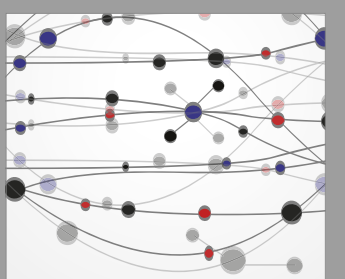

The Scientific World Journal

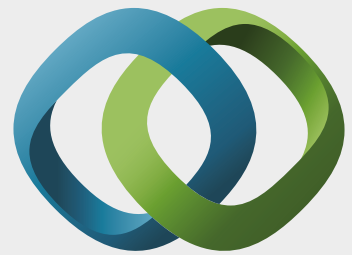

\section{Hindawi}

Submit your manuscripts at

https://www.hindawi.com
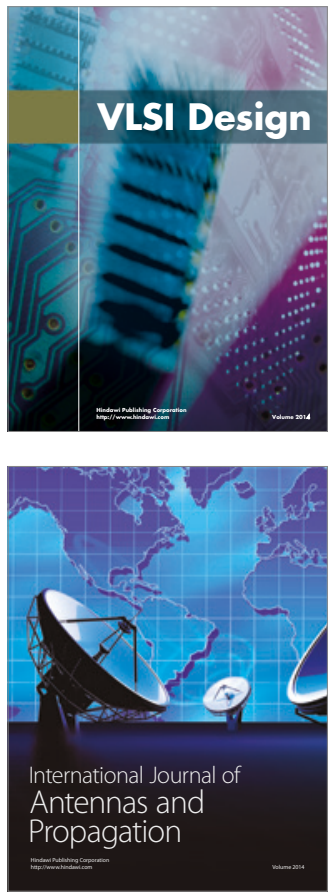

\section{Rotating}

Machinery
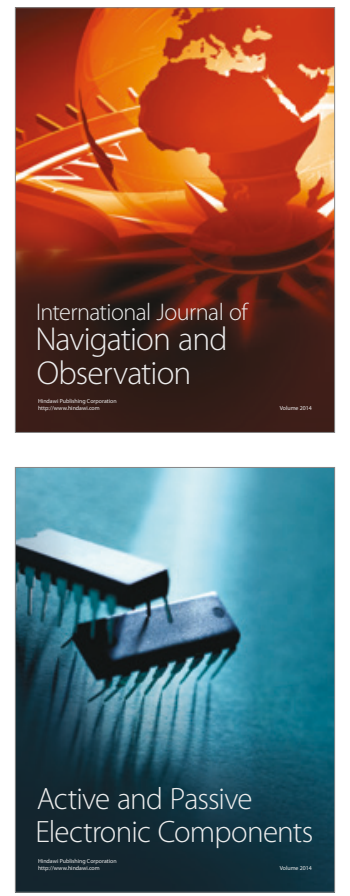
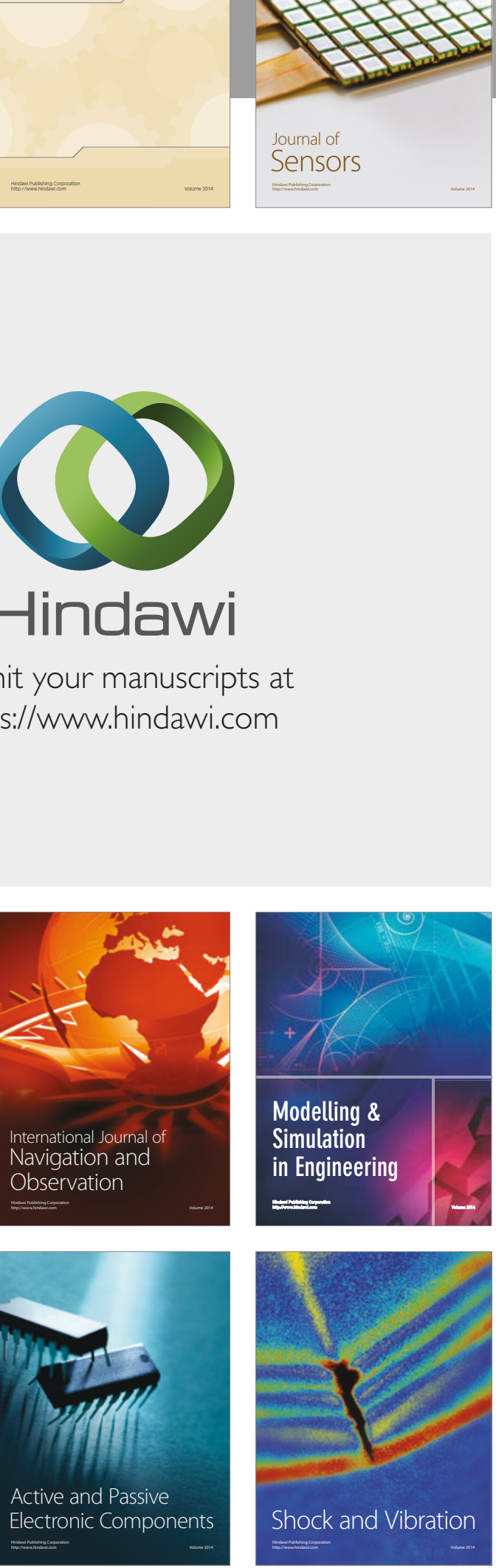
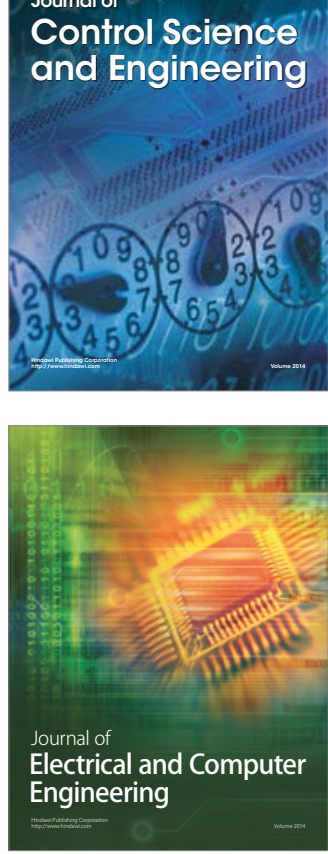

Distributed

Journal of

Control Science

and Engineering
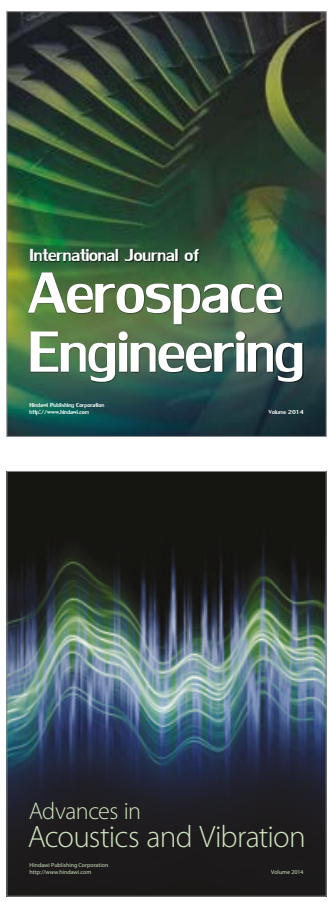

Sensor Networks 